\title{
Canadian Oncology \\ Nursing Journal
}

Revue canadienne

de soins infirmiers

en oncologie

Volume 30, Issue 1 - Winter 2020

elSSN: 2368-8076 


\title{
Optimisation du transfert des connaissances en soins palliatifs pédiatriques : utilisation d'une formation web
}

\author{
par Marie-Charel Nadeau, Karine Bilodeau et Lysanne Daoust
}

\section{INTRODUCTION}

L es infirmières ont un rôle central à jouer dans la prestaLtion de soins palliatifs, car elles influent sur leur qualité (Montgomery, Sawin et Hendricks-Ferguson, 2016). Elles sont des professionnelles de choix pour évaluer les symptômes de la maladie ou de la détresse psychologique, assurer une gestion efficace des symptômes ainsi qu'accompagner le patient et sa famille lors des prises de décisions sur les soins, que ce soit avec la clientèle adulte ou pédiatrique (Contro, Larson, Scofield, Sourkes et Cohen, 2004). Une pratique optimale en soins palliatifs peut prévenir ou soulager la souffrance de patients en fin de vie de tous âges, surtout si elle inclut l'évaluation des symptômes ainsi qu'une offre de soutien psychologique et social au patient ainsi qu'à sa famille (Qaseem et al., 2008). Bien que la majorité des soins palliatifs s'adresse à une clientèle adulte, il n'en reste pas moins que plus de 4000 enfants au Canada vivent avec une maladie incurable et ont besoin de soins palliatifs de qualité (Widger, Cadell, Davies, Siden et Steele, 2012). Cependant, des études réalisées auprès d'infirmières soulignent que celles-ci éprouvent de l'anxiété quant à la prestation de soins palliatifs pédiatriques (SPP) (Mullen, Reynolds et Larson, 2015), des difficultés à communiquer avec les familles (Montgomery et al., 2017) ainsi que des difficultés à gérer leurs émotions lors des soins de fin de vie pour un enfant (Roberts et Boyle, 2005). Selon l'hypothèse première de Contro et collaborateurs (2004), ces comportements s'expliqueraient par le manque de connaissances des infirmières en ce qui a trait aux SPP.

D’ailleurs, les écrits recensés montrent que les outils utilisés pour synthétiser et transmettre les connaissances en SPP

\section{AUTEURES}

Marie-Charel Nadeau, inf., M.Sc. Infirmière consultante en soins palliatifs, Centre intégré de santé et de services sociaux de la Montérégie-Est, SaintHyacinthe, Québec

marie-charel.nadeau@umontreal.ca

Karine Bilodeau, inf., Ph.D., CSIO(c), Professeure adjointe, Faculté des sciences infirmières, Université de Montréal, Montreal, Quebec

Auteure-ressource : C.P. 6128, Station Centre-ville, Montréal (Québec) H3C $3 \mathrm{~J} 7$

Téléphone : 514-343-6111, poste 43254 ; Fax : 514-343-2306

Courriel : Karine.bilodeau.2@umontreal.ca

Lysanne Daoust, inf., M.Sc. Infirmière clinicienne en soins palliatifs pédiatriques, Centre hospitalier universitaire Sainte-Justine, Montréal, Québec

DOI: $10.5737 / 236880763012330$ ne permettent pas aux infirmières de se renseigner rapidement et efficacement (Arenella, Yox, Eckstein et Ousley, 2010; Contro et al., 2004; Morgan, 2009; Wiener et al., 2015). L'impression de ne pas être outillées engendre alors de l'anxiété et un sentiment d'incompétence parmi les infirmières soignantes (Contro et al., 2004; Kenny, Cargil, Hamilton et Sales, 2016; Mullen et al., 2015; Smith et Gordon, 2009). Résultat : ces dernières perdent confiance en leurs capacités à prodiguer des SPP, ce qui peut altérer la qualité des soins offerts (Montgomery et al., 2016). Mentionnons que le fait de donner accès à de la formation et à des outils cliniques peut renforcer le sentiment de confiance des infirmières soignantes en ce qui touche ce type de soins (Contro et al., 2004). Cependant, les programmes de formation professionnelle sont surtout axés sur les besoins en fin de vie des adultes, et non des enfants (Association canadienne de soins palliatifs, 2006). Ainsi, il est possible de croire que le recours à une stratégie de transfert des connaissances (TC) adaptée au contexte des SPP permettrait aux infirmières d'acquérir la confiance nécessaire pour prodiguer des soins palliatifs de qualité. L'une des stratégies pour la formation en santé consiste en l'utilisation d'une formation web, car elle semble flexible, accessible et appropriée pour transmettre de l'information aux infirmières. En effet, la méta-analyse d'Arenella, Yox, Eckstein et Ousley (2010) a montré que l'utilisation des technologies de l'information (p. ex. vidéo, webinaire, apprentissage en ligne) est une stratégie très efficace pour améliorer les connaissances, les compétences et le comportement des professionnels de la santé. Aucune étude n'a présenté de conclusion sur l'utilisation de cette stratégie auprès d'infirmières en SPP précisément, mais les travaux de Morgan (2009) montrent que $66 \%$ des infirmières œuvrant en milieu hospitalier préfèrent apprendre par vidéo. À cet égard, Ruiz, Michael et Leipzig (2006) ainsi que Wiener, Weaver, Bell et Samson-Daly (2015) ajoutent que les technologies de l'information ont l'avantage de personnaliser l'apprentissage en permettant à l'infirmière d'apprendre à son rythme et de faire des liens avec ses expériences, tout en proposant une formule d'enseignement flexible. Les auteurs proposent de rendre les connaissances facilement accessibles, par exemple en les partageant sur une plateforme web (Wiener et al., 2015). Ainsi, la formation vidéo permet de concrétiser les connaissances et d'illustrer la mise en pratique de ces nouvelles connaissances. Elle a aussi l'avantage d'assurer l'accessibilité de la formation par l'intranet du centre hospitalier.

Dans le cadre d'un stage de maîtrise en sciences infirmières, une formation web a été développée selon une approche de transfert des connaissances. Le but de cet article 
est de présenter le développement et la mise à l'essai d'une formation web sur les SPP destinée aux infirmières soignantes d'unités en oncologie pédiatrique.

\section{CADRE DE RÉFÉRENCE : APPROCHE DE TRANSFERT DES CONNAISSANCES (TC) INTÉGRÉ}

Afin de créer une formation de qualité basée sur des résultats probants, une approche de TC a été utilisée (figure 1). Cette approche repose sur le modèle de TC élaboré par l'Association des infirmières et infirmiers autorisés de l'Ontario (AIIAO/RNAO) en 2012 et inspiré des travaux de Graham et al. (2006). Ce modèle, qui assure une organisation structurée de la mise en œuvre de l'intervention (création de la formation web), souligne l'importance de synthétiser et d'adapter les connaissances afin d'élaborer une stratégie de TC efficace tout en tenant compte des ressources disponibles dans le milieu ainsi que des facteurs favorables et défavorables à l'utilisation des connaissances. Le triangle au centre du modèle vient accentuer l'importance de la contribution des infirmières dans la réalisation d'une formation adaptée au milieu de soins. Dans leur étude, Grimshaw et al. (2001) corroborent l'importance de faire participer les infirmières en mentionnant que plus les apprenants sont engagés dans un projet, plus leur intérêt augmente. Plusieurs activités basées sur ce modèle ont été réalisées afin de mener à terme le projet, soit 1) la détermination du problème, 2) l'analyse des ressources du milieu, 3) l'analyse des intervenants clés, 4) l'adaptation des connaissances au contexte local, 5) l'évaluation des facteurs favorables et des obstacles à l'utilisation des connaissances, 6) la mise en œuvre de la stratégie de transfert des connaissances ainsi que 7) l'appréciation de la stratégie (formation web).

\section{MÉTHODE}

Milieu, participants et recrutement

Le projet de stage a été réalisé en collaboration avec l'unité de soins palliatifs pédiatriques du Centre hospitalier universitaire (CHU) Sainte-Justine, située à Montréal (Québec, Canada). Le secteur de l'oncologie a été ciblé, car les infirmières y prodiguent des SPP plus régulièrement que dans les autres unités. Ces dernières ont participé au projet de manière volontaire, selon leurs disponibilités $(n=30)$. Les partenaires participant au développement et à la mise à l'essai de la formation web étaient des infirmières cliniciennes en soins palliatifs $(n=2)$, des infirmières-chefs en oncologie $(n=2)$, des médecins spécialisés en soins palliatifs $(n=2)$, l'infirmière chef en soins palliatifs $(n=1)$, une conseillère-cadre en oncologie ainsi que la Direction des soins infirmiers (DSI) de l'hôpital.

\section{Étapes du développement de la formation web selon une approche de TC}

1) Détermination du problème : Dans un premier temps, comme le propose le modèle de la RNAO (2012), un problème plus précis a été cerné avec l'aide de plusieurs infirmières du milieu, lequel concernait le manque de connaissances des infirmières en SPP. Pour ce faire, deux étapes ont été réalisées : explorer les besoins en formation et cibler le besoin prioritaire.

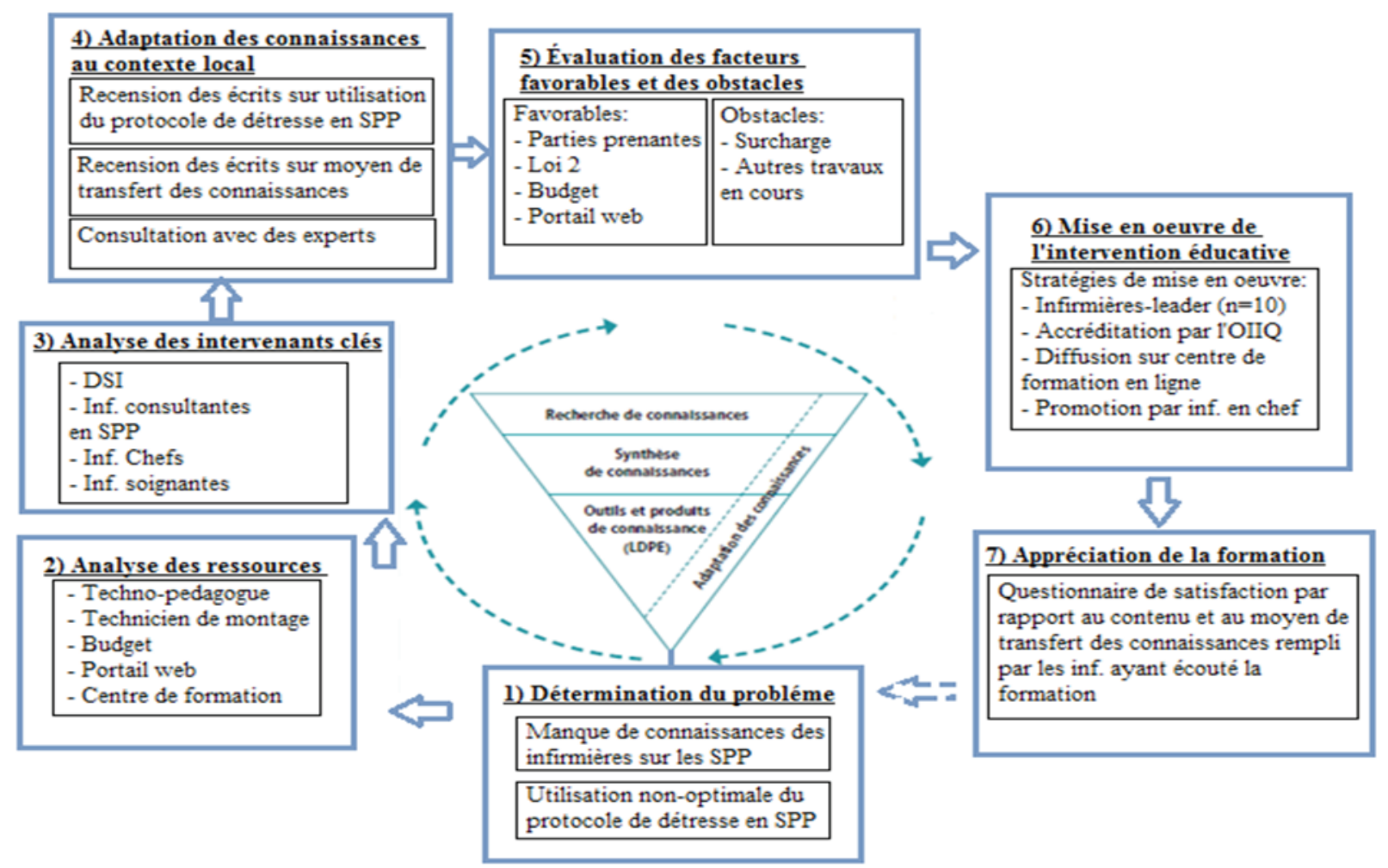

Figure 1. Approche de transfert des connaissances appliquée au développement d'une formation web. 
Exploration des besoins en formation. D'abord, des consultations auprès d'infirmières (p. ex. infirmières conseillères, chefs d'unité) ont eu lieu afin d'explorer les besoins de formation en SPP. À cela s'ajoutent des journées d'observation ayant permis de rencontrer différents professionnels et d'observer les interactions avec la clientèle pédiatrique. Ces rencontres ont fait ressortir des thèmes de formation en SPP comme la gestion de la détresse respiratoire, l'utilisation du protocole de détresse, les signes et symptômes en fin de vie, l'introduction des soins palliatifs pour les familles, la coexistence des soins curatifs et palliatifs ainsi que la transition des soins curatifs vers les soins palliatifs. Ces thèmes cadraient avec les résultats probants disponibles sur les connaissances à développer en SPP (voir tableau 1).

Détermination $d u$ besoin de formation prioritaire. Un sondage a ensuite été envoyé par courriel aux infirmières œuvrant dans l'unité d'hospitalisation en oncologie. Ce sondage, portant sur les trois quarts de travail et l'unité ambulatoire d'oncologie, les invitait à coter sur une échelle de Likert à cinq niveaux chacun des thèmes de formation établis précédemment. Le chiffre 1 correspondait à la mention «très prioritaire » et le chiffre 5 , « peu prioritaire ». Trente-deux infirmières ont participé au sondage; parmi elles, $67 \%$ ont indiqué que l'application du protocole de détresse en SPP était un besoin de formation «très prioritaire », option suivie de près par la présentation des soins palliatifs aux patients et à leur famille $(64 \%)$. Il est à noter que $67 \%$ des infirmières répondantes avaient plus de cinq années d'expérience.

2) et 3) Analyse des ressources du milieu et des intervenants clés. Une analyse des différentes ressources disponibles dans le milieu de soins a été réalisée. Un technopédagogue de l'établissement a participé au projet en contribuant à ajouter un volet interactif à la formation web, plus précisément des effets sonores, des images et des démonstrations vidéo. De plus, un budget de la DSI et de l'unité de SPP a été octroyé au projet.

4) Adaptation des connaissances au contexte local. Une recension des écrits sur l'utilisation du protocole de détresse en SPP a été réalisée afin de rédiger un scénario de formation basé sur des résultats probants. Pour ce faire, une stratégie de recherche documentaire a été élaborée en collaboration avec des bibliothécaires spécialisés en sciences de la santé, des infirmières-chefs en oncologie et des infirmières cliniciennes en SPP, le tout afin de mieux cibler les écrits les plus pertinents pour la pratique. La recherche a été menée principalement sur CINAHL, parallèlement à une recherche complémentaire sur les bases de données Google Scholar et Pubmed avec les mots clés suivants : nursing, best practices, distress protocol, palliative care et pediatrics. Une synthèse de ces résultats probants a permis de créer le contenu de la formation web et de l'organiser en cinq thèmes généraux (voir tableau 1). D'ailleurs, ce contenu a été validé par trois professionnels du milieu experts en SPP (matériel disponible dans le milieu, cohérence avec les pratiques existantes, faisabilité). Notons aussi que la formation web intègre quelques stratégies pédagogiques telles que l'utilisation d'images, d'effets sonores, de démonstrations vidéo et de mises en situation. Selon l'étude conduite par Mayer (2003), les apprenants retiennent mieux les messages quand les mots sont accompagnés d'images. De plus, Tomey (2003) avance que les étudiants apprendraient mieux par des activités qui nécessitent leur participation active telles que les mises en situation, les réflexions ou les résolutions de problèmes.

Tableau 1. Contenu de la formation web sur la détresse en SPP

\begin{tabular}{|c|c|c|c|}
\hline Thèmes & Contenu & Stratégies & Résultats probants \\
\hline $\begin{array}{l}\text { 2) Repérer une } \\
\text { détresse en pédiatrie }\end{array}$ & $\begin{array}{l}\text { La détresse sous diverses formes: } \\
\text { - Dyspnée sévère } \\
\text { - Douleur intense non soulagée par l'analgésie de base } \\
\text { - Anxiété intense et aiguë } \\
\text { - Hémorragie massive }\end{array}$ & $\begin{array}{l}\text { - Vrai ou faux } \\
\text { - Effet sonore } \\
\text { - Images } \\
\text { - Mises en situation } \\
\text { - Informations écrites }\end{array}$ & $\begin{array}{l}\text { Bidet et al. (2016), Pickstock } \\
\text { (2017), Brennan et Mazanec } \\
\text { (2011), INESSS (2017) }\end{array}$ \\
\hline $\begin{array}{l}\text { 3) Médicaments } \\
\text { utilisés }\end{array}$ & $\begin{array}{l}\text { - Midazolam } \\
\text { - Hydromorphone ou morphine } \\
\text { - Scopolamine ou glycopyrrolate }\end{array}$ & $\begin{array}{l}\text { - Vrai ou faux } \\
\text { - Images } \\
\text { - Informations écrites }\end{array}$ & $\begin{array}{l}\text { Brennan et al. (2011), Wilson } \\
\text { et al. (2015), Smothers et Beck } \\
\text { (2012), NICE (2017) }\end{array}$ \\
\hline 5) Rôles de l'infirmière & $\begin{array}{l}\text { - Observation } \\
\text { - Évaluation } \\
\text { - Documentation }\end{array}$ & $\begin{array}{l}\text { - Images } \\
\text { - Vrai ou faux } \\
\text { - Informations écrites }\end{array}$ & $\begin{array}{l}\text { Bidet et al. (2016), Bloomer et al. } \\
\text { (2015), Bobb (2016), INESSS } \\
\text { (2017) }\end{array}$ \\
\hline
\end{tabular}


5) Évaluation des facteurs favorables et des obstacles. Plusieurs facteurs favorables ont contribué à la réalisation de l'intervention éducative. D'abord, la collaboration avec plusieurs parties prenantes importantes dans le projet - comme les infirmières-chefs, les infirmières soignantes, la DSI ainsi que l'équipe de consultants en SPP - a permis de développer un produit fiable, valide et adapté au contexte de soins ainsi que d'en faire la promotion. Par ailleurs, le budget prévu par l'établissement pour l'intervention a assuré la création d'un produit de qualité. Par la suite, après l'entrée en vigueur de la Loi concernant les soins de fin de vie, les établissements de santé ont été tenus d'instaurer des mesures favorisant une offre de soins palliatifs optimale. Finalement, mentionnons la présence d'un portail web doté d'un centre de formation en ligne accessible aux infirmières. Comme le principal obstacle tenait à la surcharge de travail des infirmières soignantes, la formation devait être courte. Un onglet « soins palliatifs » a d'ailleurs été créé dans l'intranet du centre hospitalier, permettant aux infirmières d'y trouver la formation facilement durant les trois quarts de travail.

6) Mise en œuvre de la stratégie de transfert des connaissances. Les infirmières-chefs des départements d'oncologie ont été invitées à regarder la formation afin de pouvoir la promouvoir auprès des infirmières de leur département respectif. En effet, les infirmières gestionnaires peuvent influencer positivement les individus et leur organisation par l'utilisation de résultats issus de la recherche (Rycroft-Malone et al., 2002). De plus, 10 infirmières leaders, reconnues comme « championnes » dans les unités d'hospitalisation et de soins ambulatoires en oncologie, ont été sélectionnées pour participer à la formation. Cette stratégie visait la transmission des connaissances acquises à leurs collègues et la promotion de la formation web. Notons enfin une dernière stratégie contribuant au TC : l'accréditation de la formation par l'Ordre des infirmières et infirmiers du Québec (OIIQ) pour une heure de crédit de formation continue. En effet, Straus, Tetroe et Graham (2013) appuient cette stratégie en avançant que l'accréditation est favorable au TC.

7) Appréciation de la formation web. Un court questionnaire d'appréciation a été intégré à la fin de la formation web (voir tableau 2). Ce questionnaire, conçu spécifiquement pour le projet, n'a pas été validé et comprend des questions fermées se répondant par oui ou non et laissant place à des commentaires ou suggestions pour chacun des points. Les résultats permettront de déterminer si la formation web est un moyen efficace de TC pour d'éventuels projets en soins palliatifs et en oncologie.

\section{RÉSULTATS}

$\mathrm{Au}$ total, dix infirmières travaillant dans les départements d'hospitalisation et de soins ambulatoires en oncologie ont visionné la formation web. Cependant, seulement cinq d'entre elles ont répondu au questionnaire de satisfaction. Après le visionnement, elles ont toutes dit se sentir mieux préparées à renseigner les parents sur le protocole de détresse, à reconnaître une détresse ainsi qu'à appliquer le protocole (au

\begin{tabular}{|c|c|c|}
\hline Questions & $\begin{array}{l}\text { Nombre de } \\
\text { participants } \\
\text { avec } \\
\text { réponse } \\
\text { " oui " }\end{array}$ & $\begin{array}{l}\text { Exemple de } \\
\text { commentaires }\end{array}$ \\
\hline $\begin{array}{l}\text { Croyez-vous que cette } \\
\text { formation vous a préparé } \\
\text { adéquatement à être } \\
\text { capable de renseigner } \\
\text { adéquatement les parents } \\
\text { sur les indications du } \\
\text { protocole de détresse? }\end{array}$ & 4 & $\begin{array}{l}\text { "J'aurais aimé } \\
\text { avoir davantage } \\
\text { d'information pour la } \\
\text { famille concernant le } \\
\text { protocole." }\end{array}$ \\
\hline $\begin{array}{l}\text { Croyez-vous que } \\
\text { cette formation vous a } \\
\text { préparé adéquatement à } \\
\text { reconnaître une détresse } \\
\text { en pédiatrie? }\end{array}$ & 5 & $\begin{array}{l}\text { "Cela m'a permis de } \\
\text { bien comprendre les } \\
\text { situations de détresse et } \\
\text { comment appliquer le } \\
\text { protocole. " }\end{array}$ \\
\hline $\begin{array}{l}\text { Croyez-vous que cette } \\
\text { formation vous a préparé } \\
\text { adéquatement à savoir à } \\
\text { quel moment administrer } \\
\text { le protocole de détresse? }\end{array}$ & 5 & $\begin{array}{l}\text { "Bon résumé du } \\
\text { protocole de détresse } \\
\text { pour les utilisatrices et } \\
\text { bonne formation pour } \\
\text { les nouvelles. " }\end{array}$ \\
\hline $\begin{array}{l}\text { Croyez-vous que cette } \\
\text { formation vous a préparé } \\
\text { adéquatement à savoir } \\
\text { les différentes voies du } \\
\text { protocole de détresse? }\end{array}$ & 5 & $\begin{array}{l}\text { "Je ne connaissais pas } \\
\text { toutes les modalités } \\
\text { d'administration du } \\
\text { protocole de détresse." }\end{array}$ \\
\hline $\begin{array}{l}\text { Quel(s) élément(s) } \\
\text { retenez-vous le plus de } \\
\text { cette formation? }\end{array}$ & N/A & $\begin{array}{l}\text { "Le protocole de } \\
\text { détresse nécessite un } \\
\text { très bon jugement } \\
\text { clinique infirmier. " } \\
\text { "L'évaluation des } \\
\text { symptômes de détresse } \\
\text { et l'administration." } \\
\text { "Importance de bien } \\
\text { documenter. " } \\
\text { "Qu'il ne faut pas } \\
\text { hésiter à soulager } \\
\text { rapidement. " }\end{array}$ \\
\hline $\begin{array}{l}\text { Considérez-vous que les } \\
\text { modalités de la formation } \\
\text { étaient propices aux } \\
\text { apprentissages? }\end{array}$ & 5 & $\begin{array}{l}\text { "Quelques problèmes } \\
\text { techniques, mais } \\
\text { en somme très } \\
\text { divertissante." }\end{array}$ \\
\hline $\begin{array}{l}\text { Commentaires/ } \\
\text { suggestions? }\end{array}$ & $\mathrm{N} / \mathrm{A}$ & $\begin{array}{l}\text { "J'ai aimé l'utilisation } \\
\text { de quiz pour vérifier la } \\
\text { compréhension lors de } \\
\text { la formation. " }\end{array}$ \\
\hline \multicolumn{3}{|c|}{ Légende : N/A=non applicable } \\
\hline
\end{tabular}


moment opportun et de la bonne façon). En ce qui concerne les modalités de la formation, les cinq ont affirmé que le moyen utilisé était propice aux apprentissages en SPP. Trois participantes ont dit avoir surtout retenu la partie sur l'application du protocole de détresse en cas de râles mortels chez l'enfant, une situation stressante pour l'enfant, sa famille et les professionnels de la santé. Par ailleurs, la plupart des infirmières ont indiqué avoir retenu les mythes qui ont été défaits sur l'injection de morphine à un enfant en détresse. Il est ressorti des commentaires que plusieurs des mythes entourant les SPP ont aussi été déboulonnés dans la vidéo et qu'une majorité d'infirmières aimerait plus de formations sur les SPP.

\section{DISCUSSION}

\section{Changements dans la pratique}

Après la mise à l'essai de la formation, dont le but était de transmettre des connaissances en SPP aux infirmières, les infirmières participantes ont dit se sentir mieux préparées après le visionnement pour reconnaître une détresse, déterminer le moment où appliquer le protocole de détresse ainsi qu'appliquer le protocole. Il est intéressant de mentionner qu'environ 1 enfant sur 3 vivra des épisodes de dyspnée et de douleur intense en soins palliatifs, ce qui classe ces symptômes parmi les plus courants en SPP (Vollenbroich et al., 2016). Notons aussi que ces symptômes occasionnent une souffrance pour l'enfant en soins palliatifs, en plus d'entraîner un impact à long terme sur sa famille (Lützau et al., 2012; Vollenbroich et al., 2016). Les épisodes de détresse ont tendance à accentuer les sentiments d'impuissance et d'anxiété de l'infirmière face à la condition de l'enfant malade, principalement lorsqu'elle ne se sent pas en confiance pour prodiguer les soins (Deffner et Bell, 2005). Ses connaissances influencent directement la qualité des soins offerts; en effet, il a été montré qu'une infirmière plus anxieuse limite ses contacts avec son patient et la famille, ce qui nuit surtout à l'évaluation ainsi qu'à la gestion des symptômes de détresse (Mullen et al., 2015). L'acquisition de connaissances sur le sujet pourrait donc atténuer les sentiments d'anxiété et d'incompétence en SPP et, ainsi, optimiser la qualité des soins. L'infirmière doit prendre conscience de son rôle dans l'accompagnement de la famille lorsque le protocole de détresse doit être appliqué, d'où l'importance de se renseigner sur le sujet. Il ne s'agit que d'une parcelle des connaissances lui étant nécessaires pour prodiguer des SPP en toute confiance, cette formation servant de projet d'amorce en matière de TC en SPP pour l'établissement.

Ensuite, des commentaires ont été émis sur les différents mythes présentés dans la formation. La majorité des infirmières ont affirmé que la formation avait permis de déconstruire la fausse croyance associée à l'accélération de la mort par le protocole de détresse. Selon l'étude de Bidet et al. (2016), 54 \% des répondants à un sondage sur l'utilisation du protocole de détresse croient que ce dernier n'est pas optimal, parce qu'il précipite parfois la mort. De plus, selon les infirmières de cette même étude, les trois principaux obstacles à l'application du protocole sont la peur de contrevenir aux désirs du patient ou de sa famille, la peur de précipiter le décès ainsi que la peur de mal évaluer la détresse du patient. La formation web traite entre autres des deux derniers obstacles dans l'unique but que les enfants et leurs proches puissent bénéficier de soins palliatifs d'une qualité maximale.

\section{Satisfaction des infirmières au regard de la formation}

L'amélioration de la pratique infirmière en SPP par l'utilisation adéquate du protocole de détresse peut s'expliquer par l'acquisition de connaissances chez les infirmières, à l'aide des divers moyens pédagogiques de la formation. Selon les réponses des participantes, la formation web constitue un outil propice aux apprentissages et un moyen de transfert des connaissances flexible, qui leur a permis de faire des liens concrets avec leur pratique clinique. Elles ont aussi ajouté avoir apprécié l'accessibilité de la formation, disponible en tout temps et durant tous les quarts de travail, et sa durée (35 minutes). Compte tenu des contraintes de temps inhérentes à leur travail, il était d'autant plus important de réfléchir à un format de formation web qui soit le mieux adapté possible au contexte du milieu. De manière générale, les participantes semblent satisfaites des connaissances acquises et du moyen de TC.

Bien que dix participantes aient suivi la formation, seulement cinq ont pris le temps de répondre au questionnaire de satisfaction. Ce taux de réponse peut s'expliquer par la surcharge de travail et le manque de temps, la formation ayant eu lieu pendant les heures de travail.

\section{Forces de la formation web}

Les forces de la formation web sont multiples. En ce qui a trait au processus de cocréation et de mise à l'essai de la formation, l'utilisation du modèle de TC de la RNAO (2012) a permis de structurer l'intervention éducative et d'élaborer un produit adapté au contexte de soins. Les différentes étapes mentionnées dans ce modèle ont d'abord permis la définition d'une problématique générale, soit le manque de connaissances des infirmières sur les SPP. De plus, les activités réalisées en collaboration avec les infirmières ont permis de développer une formation adaptée à un contexte de soins précis. À l'Hôpital de Stanford (en Californie), un projet semblable a été réalisé, qui a favorisé un enrichissement des connaissances des infirmières ainsi qu'une amélioration de leur attitude et de leur compétence globale pour prodiguer des soins palliatifs. Les répercussions notées concernaient l'augmentation de la qualité des soins et la diminution du sentiment d'impuissance des parents (Petersen et al., 2016).

Le format vidéo retenu compte aussi parmi les forces : l'utilisation des technologies de l'information et des communications améliore les environnements d'apprentissage en favorisant le lien entre la théorie et la pratique, même lorsque l'apprentissage s'effectue à distance (Dunn, 2000; Hall, 2009; Laferrière et Gervais, 2008). Pour les infirmières, c'est un bon moyen pour apprendre à leur rythme (Wiener et al., 2015), moyen aussi considéré comme plus flexible et accessible, favorable à l'intégration des technologies de l'information et des communications dans l'apprentissage et l'enseignement (Brown, Kirkpatrick, Mangum et Avery, 2008).

Enfin, mentionnons parmi les forces la dimension interactive de la formation. En effet, dans le domaine de 
l'enseignement, toute stratégie éducative autre que la présentation magistrale est souvent considérée comme innovante. Cependant, on parle davantage d'innovation pédagogique lorsque la stratégie d'enseignement est basée sur l'interactivité entre l'utilisateur et un système d'information dans des situations visant à améliorer la qualité des apprentissages de l'apprenant (Dubois et Giroux, 2012). Dans le cadre d'interventions éducatives interactives, on présente un contenu authentique qui encourage l'apprenant à écouter et à s'engager dans le processus d'apprentissage (Tinio, 2002). Selon Cabana et collaborateurs (1999), le manque de motivation serait un obstacle au changement de comportement en santé. Les professionnels doivent être informés, motivés et formés pour intégrer les résultats probants à leur travail quotidien (Grol et Wensing, 2004). Les formations web doivent inclure diverses stratégies (p. ex. affirmations à rectifier, mises en situation, démonstrations vidéo) afin de rendre le contenu interactif et de permettre à l'infirmière de faire des liens directs entre les enseignements et sa pratique actuelle (Arenella et al., 2010).

\section{Limites de la formation web}

D'abord, une validation des données répertoriées a été effectuée auprès de différents experts en SPP, mais pas auprès des infirmières soignantes. Leur consultation aurait pourtant été un atout dans la réalisation de l'intervention éducative et aurait favorisé une plus grande participation de ces dernières. Il est à préciser que le projet s'inscrivait dans les travaux d'une maîtrise avec stage. En raison de l'échéancier et des objectifs de formation, seule une évaluation de l'appréciation de la formation a pu être réalisée auprès de quelques infirmières. Il serait pertinent d'effectuer une évaluation formelle des effets de la formation sur l'apprentissage des infirmières et leur pratique en SPP.

Ensuite, l'utilisation d'un seul moyen de TC (non combiné à un autre moyen) peut ne pas offrir un TC optimal. En effet, l'utilisation de plusieurs interventions en TC permettrait d'englober une plus grande diversité de styles d'apprentissage, une pratique qui aurait donc plus de chances d'être efficace que les interventions isolées (Bastable, 2014; Sinclair, Kable et Levett-Jones, 2015). Selon les résultats de la revue systématique de Sinclair et al. (2015), une intervention multimodale peut être nécessaire en TC - et préférable à une seule intervention - afin de réduire les taux de rappel excessifs auprès des professionnels. De plus, bien qu'utile de par sa flexibilité et son accessibilité, la formation web n'est pas la meilleure stratégie pour inclure des réflexions et du mentorat. Les réflexions personnelles et les débriefings d'équipe assureraient l'assiduité professionnelle et l'adaptation émotionnelle (Anneser, Kunath, Krautheim et Borasio, 2014). Par exemple, les apprentissages auraient pu être renforcés en accompagnant la formation d'un retour avec les infirmières sous forme d'exercice de réflexion sur le sujet présenté. Les discussions avec d'autres professionnels - qui amènent parfois à découvrir qu'on partage des perceptions, des sentiments ou un bagage d'expériences - aident à modifier les comportements ou les attitudes, favorisent le sentiment de cohésion et réduisent l'isolement (Bastable, 2014).

\section{IMPLICATIONS POUR LA PRATIQUE ET LA FORMATION INFIRMIÈRE}

Ce projet de formation web en SPP pourra avoir plusieurs effets sur la pratique et la formation des infirmières de l'établissement. En plus de répondre à un besoin de formation, le projet pourra réduire l'anxiété liée aux SPP (Mullen et al., 2015), faciliter la communication en soins palliatifs (Montgomery et al., 2017) et contribuer à la gestion des émotions (Roberts et Boyle, 2016), et cela afin d'offrir des soins palliatifs de qualité. La formation web semble donc être un outil concret assurant un transfert des connaissances théoriques à la pratique clinique (Sinclair et al., 2015) tout en permettant le développement d'une pratique professionnelle basée sur les résultats probants. Bien que ce projet ne représente qu'une partie de la formation nécessaire en SPP, il permettra aux infirmières soignantes d'acquérir de l'autonomie et de gagner en confiance lors d'un épisode de détresse d'un enfant en fin de vie. Il pourra faciliter la prise en charge efficace du patient et de sa famille de même qu'offrir une meilleure qualité de soins infirmiers.

\section{CONCLUSION}

Pour conclure, soulignons qu'il est possible de contribuer à l'avancement des soins infirmiers par le développement et l'application de connaissances issues de la recherche. La création d'une formation web constitue un moyen flexible et accessible pour s'informer sur un sujet délicat tel les SPP. Les infirmières, qui ont un rôle essentiel à jouer lors de la prestation de soins palliatifs pédiatriques dans les unités de soins, semblent anxieuses à l'idée de prodiguer ce type de soins. Afin d'être reconnues, les infirmières doivent mettre à jour leurs connaissances sur les pratiques probantes en soins palliatifs pédiatriques. Elles pourront ainsi en faire la promotion et assurer une qualité de soins optimale auprès des patients et de leur famille.

\section{REMERCIEMENTS}

Nous voulons remercier toutes les personnes du CHU SainteJustine ayant participé au projet. Nous aimerions aussi souligner l'appui financier sous forme de bourses d'études décernées à MCN par l'Ordre régional des infirmières et infirmiers de la Montérégie (ORIIM) et la Faculté des sciences infirmières de l'Université de Montréal. 


\section{RÉFÉRENCES}

Anneser, J., Kunath, N., Krautheim, V., \& Borasio, G. D. (2014). Needs, expectations, and concerns of medical students regarding end-oflife issues before the introduction of a mandatory undergraduate palliative care curriculum. Journal of Palliative Medicine, 17(11), 1201-1205.

Arenella, C., Yox, S., Eckstein, D. S., \& Ousley, A. (2010). Expanding the reach of a cancer palliative care curriculum through web-based dissemination: A public-private collaboration. Journal of Cancer Education, 25(3), 418-421.

Association canadienne de soins palliatifs. (2006). Soins palliatifs pédiatriques : Principes directeurs et normes de pratique. http://acsp. net/media/34584/Normes_pediatriques-soins_palliatifs_31_ mars_2006.pdf

Bastable, S. B. (2014). Nurse as educator: Principles of teaching and learning for nursing practice (4th ed.). Burlington: MA. Jones \& Bartlett Learning.

Bidet, G., Daoust, L., Duval, M., Ducruet, T., Toledano, B., Humbert, N., \& Gauvin, F. (2016). An order protocol for respiratory distress/ acute pain crisis in pediatric palliative care patients: medical and nursing staff perceptions. Journal of Palliative Medicine, 19(3), 306313. doi:10.1089/jpm.2015.0100

Bloomer, M., O'Connor, M., Copnell B., \& Endacott, R. (2015). Nursing care for the families of the dying child/infant in paediatric and neonatal ICU: Nurses' emotional talk and sources of discomfort. A mixed methods study. Australian Critical Care, 28, 87-92.

Bobb, B. (2016). Dyspnea and delirium at the end of life. Clinical Journal of oncology Nursing, 20(3), 244-246.

Brennan, C., \& Mazanec, P. (2011). Dyspnea management across the palliative care continuum. Journal of Hospice and Palliative Nursing, 13(3), 130-139. doi:10.1097/NJH.0b013e3182148314

Brown, S. T., Kirkpatrick, M. K., Mangum, D., \& Avery, J. (2008). A review of narrative pedagogy strategies to transform traditional nursing education. Journal of Nursing Education, 47(6), 283-286.

Cabana, M. D., Rand, C. S., Powe, N. R., Wu, A. W., Wilson, M.H ., Abboud, P. A., \& Rubin, H.R . (1999). Why don't physicians follow clinical practice guidelines? A framework for improvement. Journal of American Medical Association, 282(15), 1458-1465.

Contro, N. A., Larson, J., Scofield, S., Sourkes, B., \& Cohen, H. J. (2004). Hospital staff and family perspectives regarding quality of pediatric palliative care. American Academy of Pediatrics, 114(5), 1248-1252

Deffner, J., \& Bell, S. (2005). Nurses' death anxiety, comfort level during communication with patients and families regarding death, and exposure to communication education: A quantitative study. Journal for Nurses in Professional Development, 21, 19-23.

Dubois, S., \& Giroux, M.-N. (2012). L'innovation pédagogique chez les infirmières dans un contexte de début d'expérience professionnelle. Recherche en soins infirmiers, 111, 71-80. doi:10.3917/rsi.111.0071

Dunn, N. (2000). L'innovation par la technologie et l'apprentissage : le défi d'un programme d'études à forte composante technologique : Réseau des Écoles Innovatrices (REI) du programme canadien Rescol.

Gouvernement du Canada. (2006). Planification préalable des soins : le projet sur le glossaire - Rapport final. https://www.canada.ca/fr/ sante-canada/services/systeme-soins-sante/rapports-publications/ soins-palliatifs / planification-prealable-soins-projet-glossairerapport-final.html

Graham, I. D., Logan, J., Harrison, M. B., Straus, S. E., Tetroe, J., Caswell, W., \& Robinson, N. (2006). Lost in knowledge translation: Time for a map? Journal of Continuing Education in the Health Professions, 26(1), 13-24.
Grimshaw, J. M., Shirran, L., Thomas, R., Mowatt, G., Fraser, C. Bero, L., Grilli, R., Harvey, E., Oxman, A., \& O’Brien, M.A. (2001). Changing provider behavior: An overview of systematic reviews of interventions. Medical Care, 39(8), 112-145.

Grol, R., \& Wensing, M. (2004). What drives change? Barriers to and incentives for achieving evidence-based practice. Medical Journal of Australia, 180, 57-60.

Hall, W. A. (2009). Whither nursing education? Possibilities, panaceas, and problems. Nurse Education Today, 29, 268-275.

Institut national d'excellence en santé et services sociaux (INESSS). (2017). Protocol medical. https://www.inesss.qc.ca/fileadmin/doc/ INESSS/Ordonnances_collectives/Detresse/INESSS-Protocolemedical-Detresse.pdf

Kenny, G., Cargil, J., Hamilton, C., \& Sales, R. (2016). Improving and validating children's nurses communication skills with standardized patients in end of life care. Journal of Child Health Care, 20(2), 145-152. doi:10.1177/1367493514555588

LégisQuébec, (2019). Loi concernant les soins de fin de vie. Récupéré à http://legisquebec.gouv.qc.ca/en/ShowDoc/ cs/S-32.0001?langCont=fr

Lützau, P., Otto, M., Hechler, T., Metzing, S., Wolfe, J., \& Zernikow, B. (2012). Children dying from cancer: parents' perspectives on symptoms, quality of life, characteristics of death, and end-of-life decisions. Journal of Palliative Care, 28(4), 274-281.

Mayer, R. E. (2003). The promise of multimedia learning: using the same instructional design methods across different media. Elsevier, 13(2), 125-139.

Montgomery, K., Sawin, K. J., \& Hendricks-Ferguson, V. L. (2016). Experiences of pediatric oncology patients and their parents at end of life: A systematic review. Journal of pediatric oncology nursing, 33(2), 85-104. doi:10.1177/1043454215589715

Montgomery, K., Sawin, K., \& Hendricks-Ferguson, V. (2017). Communication during palliative care and end of life. Cancer Nursing, 40(2), 47-57.

Morgan, D. (2009). Caring for dying children: Assessing the needs of the pediatric palliative care nurse. Journal of Pediatric Nursing, 35(2), 86-90.

Mullen, J. E., Reynolds, M. R., \& Larson, J. S. (2015). Caring for pediatric patients' families at the child's end of life. Journal of Pediatric Care, 35(6), 4655. doi:http://dx.doi.org/10.4037/ ccn2015614

National Institute for Health and Care Excellence (NICE). (2017). Endof-life care for people living with life-limiting condition. Retrieved from https://www.nice.org.uk/guidance/qs13

Petersen, C., Callahan, M. F., McCarthy, D. O., Hughes, R. G., WhiteTraut, R., \& Bansal, N. K. (2016). An online educational program improves pediatric oncology nurses' knowledge, attitudes, and spiritual care competence. Journal of Pediatric Oncology Nursing, 34(2), 130-139. doi:10.1177/1043454216646542

Pickstock, S. (2017). Breathlessness at end of life: What community nurses should know. Journal of Cancer Nursing, 31(5), 74-77.

Qaseem A., Snow, V., Shekelle, P., Casey, D. E., Cross, J. T., Owens, D. K.,et al. (2008). Evidence-based interventions to improve the palliative care of pain, dyspnea, and depression at the end of life: A clinical practice guideline from the American College of Physicians. Annals Intern Medicine, 148(2), 141-146.

Registered Nurses Association of Ontario (RNAO) (2012). Toolkit: Implementation of best practice guidelines (2nd ed.). Toronto, Registered Nurses' Association of Ontario. 
Roberts, K. E., \& Boyle, L. A. (2005). End-of-life education in the pediatric intensive care unit. Critical Care Nurse, 25, 51-57.

Ruiz, J., Michael, J., \& Leipzig, R. (2006). The impact of e-learning in medical education academic medicine. Journal of Cancer Education, 81(3), 207-212.

Rycroft-Malone, J., Kitson, A., Harvey, G., McCormack, B., Seers, K., Titchen, A., \& Estabrooks, C. (2002). Ingredients for change: Revisiting a conceptual framework. Quality and Safety in Health Care, 11(2), 174-180.

Sinclair, P., Kable, A., \& Levett-Jones, T. (2015). The effectiveness of internet-based e-learning on clinician behavior and patient outcomes: A systematic review protocol. Journal of Continuing Education in the Health Professions, 13(1), 52-64. doi:10.11124/ jbisrir-2015-1919

Smothers, A., \& Buck, J. (2012). An evaluation of a practice change to increase understanding of the use of nonpharmacological interventions for the treatment of dyspnea in hospice patients. Journal of Hospice and Palliative Nursing, 14(8), 524-532. doi:10.1097/NJH.0b013e31825c7b2a

Straus, S., Tetroe, J., \& Graham, I. D. (Eds.). (2013). Knowledge translation in health care: moving from evidence to practice. Toronto, ON. John Wiley \& Sons.
Tinio, V. (2002). ICT in education. Retrieved from http://wikieducator. org/images/f/ff/Eprimer-edu_ICT_in_Education.pdf

Tomey, A. M. (2003). Learning with cases. Journal of Continuing Education in Nursing, 34, 34-38.

Vollenbroich, R., Borasio, G. D., Duroux, A., Grasser, M., Brandstätter, M., \& Führer, M. (2016). Listening to parents: The role of symptom perception in pediatric palliative home care. Palliative Support Care, 14, 13-19. doi:10.1017/S1478951515000462

Widger K., Cadell S., Davies B., Siden H., Steele R. (2012) Pediatric Palliative Care in Canada. In: Knapp C., Madden V., Fowler-Kerry S. (eds) Pediatric Palliative Care: Global Perspectives. Springer, Dordrecht. doi:10.1007/978-94-007-2570-6_17

Wiener, L., Weaver, M. S., Bell, C., \& Sansom-Daly, U. M. (2015). Threading the cloak: Palliative care education for care providers of adolescents and young adults with cancer. Journal of Clinical Oncology Adolescent and Young Adults, 5, 1-18. doi:10.2147/COAYA. S49176

Wilson, E., Morbey, H., Brown, J., Payne, S., Seale, C., \& Seymour, J. (2015). Administering anticipatory medications in end-of-life care: A qualitative study of nursing practice in the community and in nursing homes. Journal of Palliative Medicine, 29, 60-70. doi:10.1177/0269216314543042. 